\title{
Realidad actual de la enseñanza en inglés en la educación superior de Ecuador
}

\author{
Current reality of teaching in English in higher education in Ecuador
}

\section{Atual realidade do ensino de inglês no ensino superior no Equador}

\author{
Shirley R. Ponce-Merino I \\ shaley_88@hotmail.com \\ Marcia E. Parrales-Poveda ${ }^{\text {II }}$ \\ marciapapo03@hotmail.com \\ Shirley Y. Baque-Arteaga III \\ yadi558@hotmail.es \\ María L. Parrales-Poveda IV \\ maria.parrales@unesum.edu.ec
}

Recibido: 10 de enero de 2019 *Aceptado: 08 de febrero de 2019 * Publicado: 05 de abril de 2019

I. Magister en Enseñanza del Idioma Ingles; Magister en Docencia Universitaria e Investigación Educativa; Diplomado en Autoevaluación y Acreditación Universitaria; Licenciada en Ciencias de la Educación ESPEC. Inglés; Profesora de Segunda Enseñanza; Docente Universidad Estatal del Sur de Manabí, Manabí, Ecuador.

II. Licenciada en la Especialización de Terapia de Lenguaje; Tecnólogo Medico en la Especialización de Terapia de Lenguaje; Docente de la Escuela Especial Ana Luz Solís, Jipijapa, Ecuador.

III. Ingeniera Comercial mención Comercio Exterior., Universidad Estatal del Sur de Manabí, Manabí, Ecuador.

IV. Doctor en Ciencias Pedagógicas; Magister en Administración de Empresas; Magister en Docencia Universitaria e Investigación Educativa; Diplomado en Autoevaluación y Acreditación Universitaria; Economista; Docente Titular Universidad Estatal del Sur de Manabí, Manabí, Ecuador. 


\title{
Resumen
}

En Ecuador, el sistema de educación pública introdujo una carga horaria específica para la lengua extranjera desde segundo a séptimo grado de educación general básica, en los sub niveles: elemental y media, establecido por el Ministerio de Educación en la Reforma Curricular de fecha 17 de Febrero de 2016. Para la Escuela de Idiomas de la Universidad de las Américas plantea que las brechas existentes en el acceso a una educación que garantice el aprendizaje del inglés se debe a que pocos colegios lo ofrecen en sus pensum de estudios en forma asertiva, la falta de preparación de los docentes, la continuidad y falta de dinamismo que requiere dominar una nueva lengua. Al ingresar los estudiantes a las Instituciones de Educación Superior se enfrentan a grandes problemas en materia de competencia lingüística que deben alcanzar para graduarse. La falta de continuidad y motivación son una de las batallas a combatir para poder mejorar. El inglés debe ser visto como una herramienta de primer orden tanto para los estudiantes como los profesores., la edad de inicio del estudio de un segundo idioma no influye en forma determinante para su aprendizaje, otro factor clave es el uso del idioma en la cotidianidad y reforzar lo aprendido a través de viajes, libros, música, cultura, visita, grupos que manejen el idioma etc. Ecuador ingresó al ranking de las mejores universidades de América Latina con la Universidad de San Francisco de Quito (USFQ) y la Escuela Politécnica Nacional. Ambas universidades alcanzaron puntajes altos debido a su influencia investigativa (citas en publicaciones académicas). La USFQ está a la cabeza de la región en este parámetro y proyección internacional. No obstante, estas universidades deben mejorar sus esfuerzos en enseñanza, en entornos investigativos y en la transferencia del conocimiento. La metodología usada es descriptiva, con un enfoque documental, es decir, revisar fuentes disponibles en la red, como google académico, con contenido oportuno, actualizado y relevante desde el punto de vista científico que enriquezca el análisis del tema planteado en este artículo.

Palabras claves: Universidad; Inglés; Práctica; Cotidiano; Mejores Empleos; Inclusión; Docentes Altamente Capacitados; Inversión.

\begin{abstract}
In Ecuador, the public education system introduced a specific timetable for the foreign language from the second to seventh grade of basic general education, in the elementary and middle levels,
\end{abstract}


established by the Ministry of Education in the Curriculum Reform of February 17. 2016. For the School of Languages of the University of the Americas, it is proposed that the existing gaps in access to an education that guarantees the learning of English is due to the fact that few schools offer it in their study curriculum in an assertive way, the lack of teacher preparation, the continuity and lack of dynamism required to master a new language. When students enter Higher Education Institutions they face major problems in terms of linguistic competence that they must achieve in order to graduate. The lack of continuity and motivation are one of the battles to fight to improve. English must be seen as a first-rate tool for both students and teachers., the age of beginning the study of a second language does not influence in a determining way for their learning, another key factor is the use of the language in everyday life and reinforce what they have learned through travel, books, music, culture, visit, groups that handle the language etc. Ecuador entered the ranking of the best universities in Latin America with the University of San Francisco de Quito (USFQ) and the National Polytechnic School. Both universities achieved high scores due to their investigative influence (citations in academic publications). The USFQ is at the head of the region in this parameter and international projection. However, these universities must improve their efforts in teaching, in research environments and in the transfer of knowledge. The methodology used is descriptive, with a documentary approach, that is, to review sources available on the web, such as academic google, with timely, updated and relevant content from a scientific point of view that enriches the analysis of the topic raised in this article.

Key words: Universidad; Inglés; Práctica; Cotidiano; Mejores Empleos; Inclusión,; Docentes Altamente Capacitados; Inversión.

\section{Resumo}

No Equador, o sistema público de educação introduziu um calendário específico para a língua estrangeira do segundo ao sétimo ano do ensino geral básico, nos níveis elementar e médio, estabelecido pelo Ministério da Educação na Reforma Curricular de 17 de fevereiro de 2016. Na Escola de Línguas da Universidade das Américas, propõe-se que as lacunas existentes no acesso a uma educação que garanta o aprendizado do inglês se devam ao fato de que poucas escolas o oferecem em seu currículo de estudo de forma assertiva, a falta de preparação de professores, a continuidade e falta de dinamismo necessário para dominar uma nova língua. Quando os estudantes 
ingressam em Instituições de Ensino Superior, enfrentam grandes problemas em termos de competência lingüística que precisam alcançar para se formarem. A falta de continuidade e motivação é uma das batalhas para lutar para melhorar. $\mathrm{O}$ inglês deve ser visto como uma ferramenta de primeira classe para alunos e professores. , a idade de iniciar o estudo de uma segunda língua não influencia de forma determinante para sua aprendizagem, outro fator chave é o uso da linguagem na vida cotidiana e reforçar o que aprenderam através de viagens, livros, música, cultura, visita, grupos que lidam com a língua etc. O Equador entrou no ranking das melhores universidades da América Latina com a Universidade de São Francisco de Quito (USFQ) e a Escola Politécnica Nacional. Ambas as universidades alcançaram altas pontuações devido à sua influência investigativa (citações em publicações acadêmicas). O USFQ está na cabeça da região neste parâmetro e projeção internacional. No entanto, essas universidades devem melhorar seus esforços no ensino, nos ambientes de pesquisa e na transferência de conhecimento. A metodologia utilizada é descritiva, com uma abordagem documental, ou seja, para revisar fontes disponíveis na web, como o google acadêmico, com conteúdo oportuno, atualizado e relevante de um ponto de vista científico que enriquece a análise do tema levantado neste artigo. artigo.

Palavras chave: Universidade; Inglês; Prática Diariamente; Melhores empregos; Inclusão; Professores altamente qualificados; Investimento.

\section{Introducción.}

El desarrollo de las naciones modernas se logra de la mano de políticas e iniciativas orientadas hacia el mejoramiento de la calidad de vida de sus habitantes mediante la puesta en marcha de distintos proyectos en educación, salud y seguridad entre otros. Un pilar que sustenta en forma sólida la mejora de la calidad de vida de la población es el desarrollo profesional, es decir, las oportunidades otorgadas a la población para su crecimiento académico y laboral.

El aprendizaje del idioma inglés cobra cada vez mayor fuerza en el mundo. El inglés es el idioma de la tecnología, del comercio y de las ciencias por lo que ciudadanos más capacitados en otras lenguas optarán por mejores empleos y remuneraciones. Esto ha sido tendencia en América 
Latina: Méjico, Colombia, Chile, Brasil, Argentina, Ecuador, Costa Rica, Uruguay, Paraguay y Perú, han implementado políticas públicas encaminadas a promover la enseñanza del inglés en sus respectivos países (Santana, 2016).

Muchas empresas crecen a nivel global, el idioma inglés se ha convertido en un medio para compartir información, establecer conversaciones y relaciones con personas alrededor del mundo permitiendo crear y cerrar negocios. Existen más de 50 países alrededor del mundo en los que se habla inglés, las opciones de trabajo en diversas partes del mundo son mayores que con cualquier otro idioma.

Resulta fácil aprender este idioma, el alfabeto del inglés es latino, corto y su gramática es sencilla, los verbos son fáciles de conjugar. La pregunta de rigor es por qué razón se estudia en educación primaria y secundaria por muchos años pero el resultado es que los estudiantes llegan a la Universidad con un conocimiento muy débil o nulo del idioma. Los que obtienen mejor desempeño en la educación superior son egresados de centros educativos privados o que invierten en cursos de idiomas particulares, abriendo un abanico de oportunidades en materia laboral para estos jóvenes.

A continuación se ahondará en posibles respuestas a las razones de esa discrepancia entre los estudios del idioma inglés y el rendimiento de los estudiantes al desarrollar la competencia del inglés para los estudiantes ecuatorianos y Latinoamericanos. El éxito en la educación superior radica no solo en obtener el diploma universitario sino en alcanzar un excelente empleo y calidad de vida.

\section{Metodología.}

Esta investigación está dirigida al estudio de la Realidad actual de la enseñanza en inglés en la educación superior de Ecuador. Para realizarlo se usó una metodología descriptiva, con un

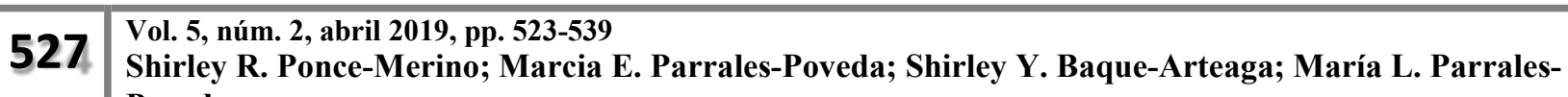
Poveda
} 
enfoque documental, es decir, revisar fuentes disponibles en la red, como google académico, con contenido oportuno y relevante desde el punto de vista científico para dar respuesta a lo tratado en el presente artículo y que sirvan de inspiración para realizar otros proyectos. Las fuentes consultadas pueden ser estudiadas al final, en la bibliografía.

\section{Resumen.}

En Ecuador, el sistema de educación pública introdujo una carga horaria específica para la lengua extranjera desde segundo a séptimo grado de educación general básica, en los sub niveles elemental y media establecido por el Ministerio de Educación en la Reforma Curricular de fecha 17 de Febrero de 2016. Antes de esta fecha los estudiantes no recibían clases de inglés de manera obligatoria en su carga académica.

El Instituto de Idiomas de la Universidad Salesiana de Ecuador realizó un análisis sobre el nivel de inglés que poseen los estudiantes, en cada ciclo estudian entre 5.800 y 6.200 alumnos. De ellos, el $5 \%$ desconoce ese idioma en su totalidad mientras que un $50 \%$ lo maneja de forma intermedia a baja y el restante (45\%) posee un buen nivel, son estudiantes provenientes de colegios que enseñan con éxito el idioma inglés o tomaron clases y cursos particulares en centros especializados (P. Armas, 2016).

Para la Escuela de Idiomas de la Universidad de las Américas plantea que las brechas existentes en el acceso a una educación que garantice el aprendizaje del inglés se debe principalmente a que pocos colegios lo ofrecen en sus pensum de estudios en forma asertiva, la falta de preparación de los docentes, la continuidad y el dinamismo que requiere dominar una nueva lengua. 
Al ingresar los estudiantes a las Instituciones de Educación Superior se enfrentan a grandes problemas en materia de competencia lingüística que deben alcanzar para graduarse. La falta de continuidad y motivación son una de las batallas a combatir para poder mejorar. El inglés debe ser visto como una herramienta de primer orden tanto para los estudiantes como los profesores, a los primeros su dominio les garantizará mejores empleos y remuneraciones a futuro a los segundo el reto abarca mejores estrategias para su enseñanza con una audiencia en desigualdad de condiciones.

En el proceso de enseñanza aprendizaje del idioma inglés los pilares fundamentales son el profesor y estudiante, de ellos dependerá el éxito del proceso, si uno de ellos falla, el aprendizaje no logra los resultados esperados. El rol del profesor en la actualidad no es protagónico sino de guía en la enseñanza, requiere docentes altamente capacitados, que maneje la metodología apropiada, diseñando estrategias apropiadas para el grupo estudiantil. Otros aspectos a considerar (Beltrán, 2017):

- La planificación de los procedimientos requiere considerar el nivel de conocimiento previo de los estudiantes, su comprensión y dominio de lo aprendido.

- Expresarse de forma clara y sencilla, reforzando las explicaciones y correcciones con los medios disponibles (dibujos, pizarra, música, etc.)

- Promocionar el uso de frases y formulas idiomáticas en inglés en la comunicación.

- Promover la capacidad del alumno para inferir el significado por medio del contexto lógico y asociaciones.

El estudiante representa los resultados del aprendizaje, el grado de competencia comunicativa que ha logrado alcanzar al final del curso o proceso. Representan el rol más activo ya que serán los responsables del nivel de desarrollo de las habilidades del idioma, corresponde una 
mayor iniciativa dentro de las aulas de clase. La madurez del estudiante influye significativamente en sus resultados, un estudiante convencido que el inglés no es una asignatura más sino una manera de accesar a mejores empleos y remuneraciones pondrá mayor esfuerzo en sus resultados.

La selección del material apropiado para ser utilizado en clases de inglés se convierte en otro elemento clave a considerar por parte de los profesores y autoridades académicas, más allá de una asignatura dentro de un pensum de estudios, se recomienda potenciar la práctica del idioma inglés no solo dentro de las aulas universitarias sino fuera de ellas, en su cotidianidad (Castro, 2016).

Los factores cognitivos incluyen la inteligencia, la aptitud y el uso de estrategias de aprendizaje. Estudios respaldan que estudiantes con inteligencia superior al promedio, aprenden más fácilmente un segundo idioma en el contexto formal de un aula. También los aprendices exitosos muestran una aptitud favorable y comprometida en esforzarse y trabajar duro en pro a la meta. El campo afectivo incluye algunos factores: empatía, autoestima, extroversión, inhibición, imitación, ansiedad entre otras potencia o dificulta el dominio de otro idioma.

Contrario a lo que se piensa comúnmente, la edad de inicio del estudio de un segundo idioma no influye en forma determinante para su aprendizaje, la ventaja que otorga aprender un idioma a primera edad obedece a una mejor pronunciación de los acentos, es decir, de forma más natural en relación a los que aprenden más adultos. Otro factor clave es el uso del idioma en la cotidianidad y reforzar lo aprendido a través de viajes, libros, música, cultura, visita, grupos que manejen el idioma etc.

El idioma ingles en Cifras 
Según un informe publicado por Education First (EF) en el año 2017, Ecuador mantiene un nivel bajo de habla y dominio del idioma en relación con otros países como Argentina (que lidera la región), seguido por República Dominicana, Costa Rica y Brasil.

Un grupo de 85.000 personas fueron evaluadas a escala nacional en Ecuador por EF, el promedio alcanzado fue de 49.32 en base a los 100 puntos, de esa muestra, los adultos de $31-40$ años alcanzaron un puntaje de 47.26 mientras que los jóvenes de 18 a 20 años un 53.57, una calificación 11\% superior con respecto a los más adultos (El Comercio, 2017).

En Costa Rica se aplica desde el año 1994 el programa Costa Rica Multilingüe (CRML), en un principio abarcó a las escuelas primarias y posteriormente en 1998 a la prescolar. Para el año 1997 se estableció el inglés como materia básica para el primer y segundo ciclo de la educación primaria, logrando un avance en la oferta académica y la cobertura en la enseñanza del idioma. Esta base le permitió a Costa Rica posicionarse hasta el año 2003 como el único país en Centroamérica que ofrecía un modelo de enseñanza del inglés en su educación primaria, logrando una cobertura del 73.7\% de la población estudiantil a nivel nacional, facilitando el dominio del idioma ingles no solo como asignatura sino en el ámbito laboral (Bonilla, Septiembre de 2012).

La calidad de la educación superior no se mide solamente por el éxito o fracaso de sus estudiantes dominen otro idioma además de su lengua materna, es importante pero no suficiente así lo determino la clasificación anual QS Latin America Rankings que se muestra a continuación:

\section{Tabla Nº1 QS Latin America Rankings 2019}
Lugar
Universidad
País 


\begin{tabular}{|c|c|c|}
\hline 1 & Pontificia Universidad Católica & Chile \\
\hline 2 & Universidad de Sao Paulo & Brasil \\
\hline 3 & Universidad Estatal de Campinas & Brasil \\
\hline$\overline{4}$ & Universidad Nacional Autónoma de México & México \\
\hline 5 & Universidad de los Andes & Colombia \\
\hline 6 & $\begin{array}{l}\text { Instituto Tecnológico y de Estudios Superiores } \\
\text { Monterrey }\end{array}$ & México \\
\hline 7 & Universidad de Chile & Chile \\
\hline 8 & Universidad de Buenos Aires & Argentina \\
\hline 9 & Universidad Federal de Río de Janeiro & Brasil \\
\hline 10 & Universidad Nacional de Colombia & Colombia \\
\hline 11 & Universidad Estatal Paulista & Brasil \\
\hline 12 & $\begin{array}{c}\text { Pontificia Universidad Católica de Río de } \\
\text { Janeiro }\end{array}$ & Brasil \\
\hline 13 & Universidad de Santiago de Chile & Chile \\
\hline 14 & Universidad de Concepción & Chile \\
\hline 15 & Universidad de Antioquia & Colombia \\
\hline 16 & Universidad Federal de Minas Gerais & Brasil \\
\hline 17 & Pontificia Universidad Javeriana & Colombia \\
\hline 18 & Universidad Federal de Río Grande del Sur & Brasil \\
\hline 19 & Universidad de Costa Rica & Costa Rica \\
\hline 20 & Universidad de La Habana & Cuba \\
\hline
\end{tabular}


Fuente: (BBC News, 2018)

El grupo QS asigna en el caso de las universidades de América Latina un puntaje a través de 8 indicadores, algunos de los considerados (BBC News, 2018):

- Reputación académica (30\%).

- Calificación que le dan generadores de empleo a la calidad de sus egresados $(20 \%)$.

- Producción de ciencia, ¿cuántos estudios genera cada universidad o qué \% se citan en otras investigaciones sus estudios.

La Pontificia Universidad Católica de Chile aparece por segundo año consecutivo en el primer lugar de posición de América Latina. La Universidad de Buenos Aires tiene un mejor lugar en la clasificación mundial que en la regional (lugar 73 mientras que la Chilena es 132). Estas discrepancias se deben a que ambas mediciones ponderan de forma diferente los indicadores usados por cada grupo. A nivel mundial por ejemplo, la reputación académica es de $40 \%$ mientras que la calificación de los empleadores es de $10 \%$.

La Pontificia Universidad Católica de Chile y la Universidad de Chile, ubicadas en los puestos 3 y 4 de la región, ubican a ese país como el segundo más destacado del índice, en el que cuenta con 15 centros de estudio entre los primeros 50, cuatro más que en 2016. Investigaciones recientes de Times Higher Education junto al Centro para la Educación Superior Global, de la University College de Londres, incluyen a Chile dentro de un grupo de siete países del mundo a los que denominan "tácticos" por considerar que son los que tienen mayores posibilidades de convertirse en las futuras estrellas de la educación superior (Camargo, 2017).

\footnotetext{
533 Vol. 5, núm. 2, abril 2019, pp. 523-539 
En cuanto a Chile, el estudio determinó que tiene unas altas tasas de participación universitaria y un alto nivel de calidad investigativa, invierte más en educación terciaria que muchos de sus vecinos. El grupo de los "tácticos" incluye a otros dos países latinoamericanos: Colombia y Argentina. Colombia cuenta con un centro de estudios entre los 10 primeros del ranking: la Universidad de los Andes, que este año quedó en el quinto puesto, luego de haber aparecido en el décimo en 2016, tiene cinco instituciones entre las primeras 50.

La producción investigativa de Colombia ha crecido a una tasa magistral $49 \%$ desde 2011. Los salarios de los investigadores son también mayores que los de muchos de sus países competidores, lo cual sugiere que es un destino atractivo para académicos. Como aspecto a mejorar, Colombia invierte menos en investigación y desarrollo y en educación terciaria que muchos de sus países vecinos y alberga una menor proporción comparativa de investigadores.

Excelentes resultados para Argentina, 2017 es el primer año que ingresa en el índice con dos centros de estudio: la Universidad Nacional de Córdoba, que quedó en el grupo ubicado entre los puestos del 26 al 30, y la Universidad Austral, que quedó entre las posiciones 61 al 70. Dentro de sus fortalezas, Argentina tiene una alta proporción de investigadores y elevadas tasas de participación universitaria e investigación de alto nivel.

El otro país de la región que ingresó por primera vez en el ranking de las mejores universidades de América Latina es Ecuador, con la Universidad de San Francisco de Quito (USFQ), ubicada entre los puestos 41 a 45; y la Escuela Politécnica Nacional, que quedó en el grupo del 61 al 70 (Camargo, 2017). Ambas universidades alcanzaron puntajes altos debido a su influencia investigativa (citas en publicaciones académicas). La USFQ está a la cabeza de la región

\footnotetext{
534 Vol. 5, núm. 2, abril 2019, pp. 523-539

Shirley R. Ponce-Merino; Marcia E. Parrales-Poveda; Shirley Y. Baque-Arteaga; María L. ParralesPoveda
} 
en este parámetro y proyección internacional. No obstante, estas universidades deben mejorar sus esfuerzos en enseñanza, en entornos investigativos y en la transferencia del conocimiento.

De acuerdo con los responsables del ranking, aunque el sector de la educación superior en América Latina está mejorando significativamente, hay riesgos y amenazas como los bajos salarios de los investigadores, la insuficiencia de fondos disponibles para investigar, el exceso de burocracia y la inadecuada definición de las políticas de investigación. El incremento de la competencia entre las distintas universidades implica que los centros deben hacer esfuerzos para no quedarse atrás (Lozada, 11 de Junio 2018).

Esto se evidencio con la Universidad de Costa Rica, (la única de ese país que aparece en el índice), entre 2016 y 2017 retrocedió del grupo correspondiente a los puestos 26 al 30 hasta colocarse entre los lugares 41 al 45. A medida que las universidades en América Latina se enfocan en el incremento de la calidad de la educación y la investigación, un alza en la participación y accesibilidad de la educación y la productividad investigativa, la competencia por los mejores académicos y estudiantes se hará cada vez más intensa.

En materia de política educativa la Secretaría Nacional de Educación Superior, Ciencia, Tecnología e Innovación (SENESCYT) de Ecuador anunció que el Programa "Enseña Inglés" entregará becas para estudios en el exterior, con el objetivo de mejorar la calidad de la enseñanza de este idioma en colegios públicos, a través de una formación mucho más acorde a las necesidades de educación del país. Su propósito es potenciar la enseñanza de este idioma en escuelas y colegios del Ecuador. Una medida que no solo busca la calidad educativa, sino también la inclusión social. En el futuro, la enseñanza de esta lengua será obligatoria desde el primer grado de escuela.

\footnotetext{
535 Vol. 5, núm. 2, abril 2019, pp. 523-539 
A partir del año 2016, el Ministerio de Educación emitió un acuerdo en el que se establece la enseñanza obligatoria de este idioma, esta estrategia permitirá que el estudiante al finalizar bachillerato alcance un nivel B1 de acuerdo al marco común europeo, lo que significa que es capaz de leer y escribir sin dificultad. El Ministerio de Educación incorporó entre 2016 y 2017, a 762 docentes angloparlantes (de habla inglesa) al sistema educativo nacional que se desempeñan en cerca de 750 instituciones con más de 800 alumnos (SENESCYT, 2017).

A continuación se muestra un gráfico que mide el avance del interés por aprender el idioma inglés no solo para los ecuatorianos sino para varios países Latinoamericanos desde el año 2007 hasta el 2015.

\section{Figura $N^{\circ} 1$. Evolución del interés por el idioma inglés}

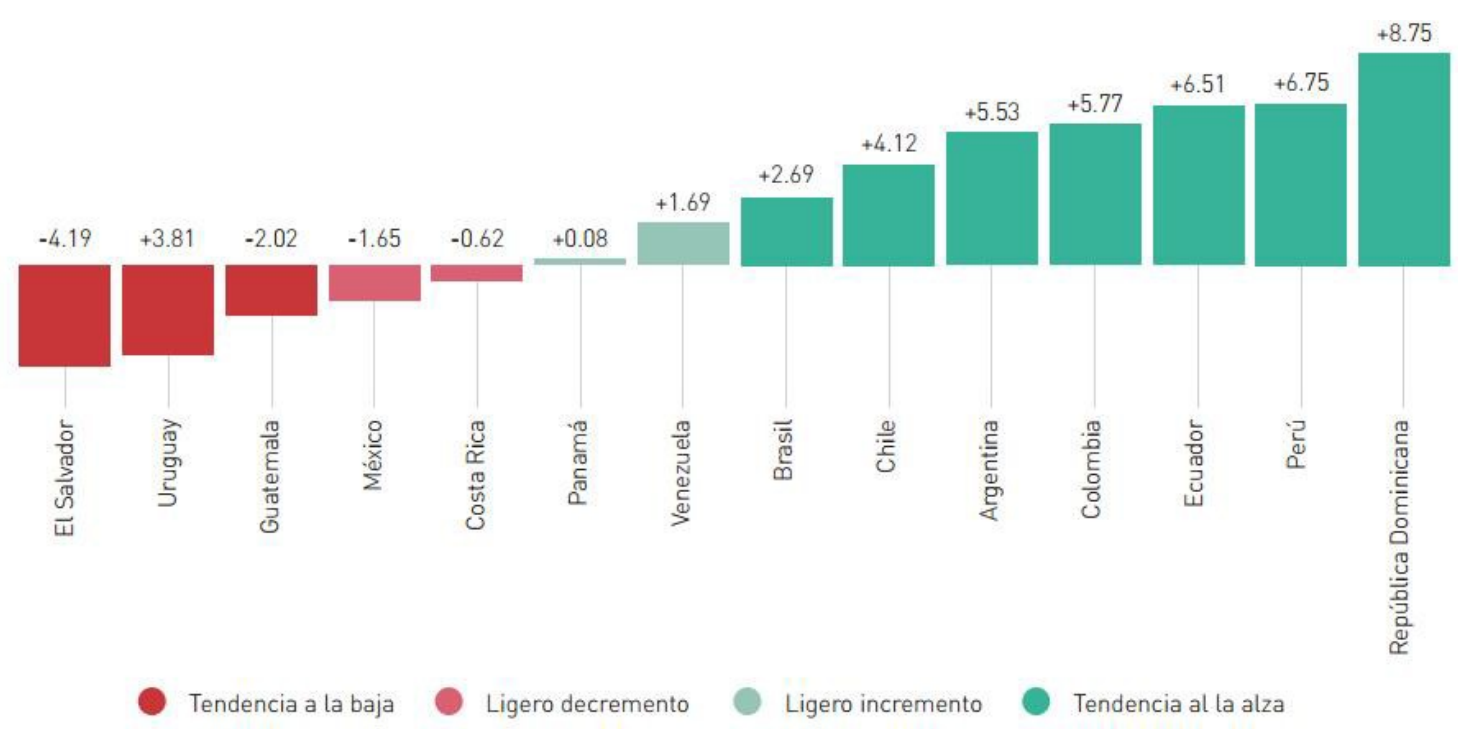

Fuente: (Beltrán, 2017) 
Otros factores que pueden influir en el aprendizaje son la conectividad con internet y el índice de facilidad para comerciar en el país; la cercanía a un país de habla inglesa no es importante, de lo contrario en los estados fronterizos se hablaría mucho inglés, lo que no ocurre, en las zonas turísticas, donde hay más necesidad, el nivel de inglés es más alto, el vínculo con la economía es evidente y directo. A pesar de que América Latina está invadida por la televisión y otros medios estadounidenses, muchos de estos vienen doblados o traducidos al idioma local, de manera que el impacto es nulo en cuestión de aprender inglés. Destacó una notoria mejoría de los niveles de inglés en la región.

Los especialistas en educación de enfoque tradicional, recomiendan la conveniencia de que este aprendizaje se haga desde los primeros años de edad, no solo para fijar mejor los conocimientos, sino para que ese niño se maneje con comodidad en otra lengua que no sea la nativa. El inglés es una herramienta vital para todos los profesionales universitarios y no universitarios en un mundo globalizado y competitivo, mientras más habilidades tenga una persona mejores oportunidades laborales en el futuro tendrá.

\section{Conclusión.}

Ecuador ha crecido en forma favorable en resultados alcanzados por varias de sus casas de Estudio a nivel superior recientemente. Estos resultados no son producto del azar sino de una política orientada a mejorar la calidad educativa desde las bases, en los sectores menos favorecidos que no pueden pagar grandes sumas de dinero en su educación.

\footnotetext{
537 Vol. 5, núm. 2, abril 2019, pp. 523-539 Shirley R. Ponce-Merino; Marcia E. Parrales-Poveda; Shirley Y. Baque-Arteaga; María L. ParralesPoveda
} 
Esa política pasa por reconocer e incluir en su programa de formación educativa el manejo de sus estudiantes del idioma inglés desde los primeros años de su formación académica desde la Reforma educativa implementada en el año 2016. La Escuela de Idiomas de la Universidad de las Américas plantea que las brechas existentes en el acceso a una educación que garantice el aprendizaje del inglés se debe principalmente a que pocos colegios lo ofrecen en sus pensum de estudios en forma asertiva, la falta de preparación de los docentes, la continuidad y falta de dinamismo que requiere dominar una nueva lengua.

$\mathrm{Al}$ ingresar los estudiantes a las Instituciones de Educación Superior se enfrentan a grandes problemas en materia de competencia lingüística que deben alcanzar para graduarse. La falta de continuidad y motivación son una de las batallas a combatir para poder mejorar. El inglés debe ser visto como una herramienta de primer orden tanto para los estudiantes como los profesores, a los primeros su dominio les garantizará mejores empleos y remuneraciones a futuro a los segundo el reto abarca mejores estrategias para su enseñanza con una audiencia en desigualdad de condiciones.

Ecuador ingresó este año por primera vez en el ranking de las mejores universidades de América Latina, con la Universidad de San Francisco de Quito (USFQ) y la Escuela Politécnica Nacional. Ambas universidades alcanzaron puntajes altos debido a su influencia investigativa (citas en publicaciones académicas). La USFQ está a la cabeza de la región en este parámetro y proyección internacional. No obstante, estas universidades deben mejorar sus esfuerzos en enseñanza, en entornos investigativos y en la transferencia del conocimiento. 
El sector de la educación superior en América Latina está mejorando significativamente, hay riesgos y amenazas como los bajos salarios de los investigadores, la insuficiencia de fondos disponibles para investigar, el exceso de burocracia y la inadecuada definición de las políticas de investigación. El incremento de la competencia entre las distintas universidades implica que los centros deben hacer esfuerzos para no quedarse atrás y perder lo alcanzado.

\section{Bibliografía}

BBC News. (2 de Noviembre de 2018). Las mejores universidades de América Latina en 2018 según el QS Latin America Ranking. Obtenido de BBC News Mundo: www.bbc.com.

Beltrán, M. (19 de Abril de 2017). El aprendizaje del idioma inglés como lengua extranjera. Obtenido de Boletín virtual Abril volumén 6 - 4: www.dialnet.unirioja.es

Bonilla, A. (Septiembre de 2012). El aprendizaje de las lenguas extranjeras como herramienta para el desarrollo humano. IV Congreso Iberoamericano de las lenguas en la Educación y la Cultura. (págs. 1 - 11). Salamanca - España: www.oei.es

Camargo, M. (20 de Julio de 2017). Estas son las mejores universidades de América Latina en 2017 (y cuáles son los 4 países que se destacan). Obtenido de BBC News Mundo: www.bbc,com

Castro, Y. (2016). Universidad Metropolitana de Ecuador. Obtenido de Perspectivas para la enseñanza del inglés en las Institusiones de Educación Superior en Ecuador.: www.pedagogía.edu.ec

El Comercio. (2017). Obtenido de El nivel de inglés en el Ecuador todavía es bajo.: www.elcomercio.com

Lozada, R. (11 de Junio 2018). Los Métodos de Enseñanza del Inglés en la Preparación Idiomática Requerida por la Universidad del Siglo XXI. Revista Científica Hallazgos $N^{\circ} 21$, volumen 3, www.revistas.pucese.edu.ec.

P. Armas. (17 de Octubre de 2016). La necesidad de aprender inglés es cada vez mayor en Ecuador. Obtenido de El Comercio: www.elcomercio.com

Santana, J. (2016). Variables que influyen sobre el aprendizaje del inglés como segunda lengua. Revista Internacional de Lenguas Extranjeras, $N^{\circ}$ 5, 79 - 94 www.core.ac.uk.

SENESCYT. (20 de Noviembre de 2017). La enseñanza del inglés en el Ecuador. Obtenido de Ecuador Universitario: www.ecuadoruniversitario.com 